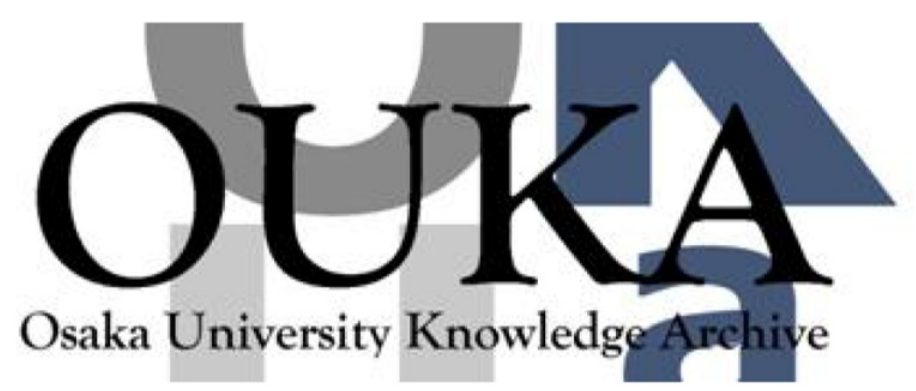

\begin{tabular}{|c|c|}
\hline Title & $\begin{array}{l}\text { Liner conformality in ionized magnetron sputter } \\
\text { metal deposition processes }\end{array}$ \\
\hline Author(s) & Hamaguchi, S.; Rossnagel, S. M. \\
\hline Citation & $\begin{array}{l}\text { Journal of Vacuum Science and Technology B: } \\
\text { Microelectronics and Nanometer Structures. } \\
\text { 14(4) p. 2603-p. } 2608\end{array}$ \\
\hline Issue Date & $1996-07$ \\
\hline oaire:version & VoR \\
\hline URL & https://hdl. hand le. net/11094/78499 \\
\hline rights & $\begin{array}{l}\text { This article may be downloaded for personal use } \\
\text { only. Any other use requires prior permission } \\
\text { of the author and AIP Publishing. This article } \\
\text { appeared in Journal of Vacuum Science \& } \\
\text { Technology B: Microelectronics and Nanometer } \\
\text { Structures Processing, Measurement, and } \\
\text { Phenomena 14, 2603 (1996) and may be found at } \\
\text { https://doi.org/10.1116/1.588993. }\end{array}$ \\
\hline Note & \\
\hline
\end{tabular}

Osaka University Knowledge Archive : OUKA

https://ir. Library. osaka-u. ac. jp/

Osaka University 


\title{
Liner conformality in ionized magnetron sputter metal deposition processes
}

\author{
S. Hamaguchia) and S. M. Rossnage ${ }^{\text {b) }}$ \\ IBM Thomas J. Watson Research Center, P.O. Box 218, Yorktown Heights, New York 10598
}

(Received 9 November 1995; accepted 19 April 1996)

\begin{abstract}
The conformality of thin metal films (liners) formed on high-aspect-ratio trench structures in ionized magnetron sputter deposition processes is studied numerically and experimentally. The numerical simulator (SHADE) used to predict the surface topography is based on the shock-tracking method for surface evolution. The simulation results are in good agreement with experimentally observed thin-film topography. It is shown that combination of direct deposition and trench-bottom resputtering results in good conformality of step coverages and the amount of the resputtering needed for the good conformality is almost independent of trench aspect ratios. (C) 1996 American Vacuum Society.
\end{abstract}

\section{INTRODUCTION}

The reliable formation of densely packed interconnect metal lines with low resistance is a challenging task in computer-chip manufacturing. The most advanced technologies currently under development require filling of interconnect lines with the aspect ratio (i.e., ratio of depth to lateral dimension) as high as three. Conventional deposition methods such as chemical vapor deposition (CVD) ${ }^{\prime}$ and collimated magnetron sputter deposition ${ }^{2-4}$ may not provide an effective means to fill such high-aspect-ratio trenches and vias in manufacturing environments.

Prior to the metal filling process into dielectric trenches and vias, lining (i.e., deposition of a thin metal layer) is often employed to provide adhesion layers or diffusion barriers for materials to be deposited in the subsequent processes. Such a thin metal film (liner) must be sufficiently conformal, i.e., uniform in thickness on sidewalls and bottoms of trenches (or vias), so that the adhesion or diffusion-barrier characteristics are also uniform on the surface.

Depositing conformal liners into high-aspect-ratio trenches and vias is also a difficult task in low-temperature processes. For metal species to reach the bottom, the metal flux incident upon the wafer surface must be anisotropic. An anisotropic flux, however, does not deposit the material onto vertical sidewalls directly. Weaker anisotropy of the incoming flux, on the other hand, may deposit the material on the sidewalls but tends to deposit more near the top edges of the trench, resulting in nonconformal deposition. This effect is routinely observed with collimated sputtering of $\mathrm{Ti}$ and $\mathrm{TiN}$ diffusion barriers. ${ }^{4}$

The ionized magnetron sputter deposition process ${ }^{5,6}$ can circumvent this dilemma by using its ability to resputter the deposited metal from the bottom of the trench. The ionized magnetron sputter deposition system is schematically depicted in Fig. 1. This is a combination of the conventional magnetron sputter system and an inductively coupled plasma generator. The metal atoms are sputtered from the magnetron

\footnotetext{
"Electronic mail: satoshi@watson.ibm.com

b)Electronic mail: rossn@watson.ibm.com
}

cathode and then ionized near the center of the chamber by the high-density Ar plasma generated by the induction coil. The bias voltage on the wafer then controls the directionality and bombardment energies of the metal and $\mathrm{Ar}$ ion fluxes incident upon the wafer surface.

If the ion bombardment energy is sufficiently high, the deposited metal on the bottom of the trench may be resputtered and then redeposited on the sidewalls. By controlling the bias voltage on the wafer, one can adjust the amount of resputtering to form a conformal metal liner over the trench structure.

\section{EXPERIMENTAL SETUP AND SIMULATION MODEL}

As shown in Fig. 1, the experimental system essentially consists of the magnetron and multiple-turn radio frequency induction (RFI) coil. The magnetron power may be typically varied up to $10 \mathrm{~kW}$ and $30 \mathrm{~kW}$ on $200 \mathrm{~mm}$ and $300 \mathrm{~mm}$ target cathodes. The RFI coil can be powered up to $3-5 \mathrm{~kW}$ at $13.56 \mathrm{MHz}$. The dc-bias voltage applied to the wafer holder may be varied up to $-200 \mathrm{~V}$ with substrate currents in the several ampere range. In typical experimental conditions, the Ar pressure is varied from 1 to $35 \mathrm{mT}$ Torr. When a large metal ion flux is desirable, the high-pressure (e.g., $35 \mathrm{mTorr}$ ) operation is employed. When the pressure is high, the sputtered-atom mean free paths decrease and thus the relative ionization level increases. Since the collision frequency in the sheath region (about $0.1 \mathrm{~mm}$ thick) above the wafer holder is still small, the increased pressure does not affect the directionality of ion fluxes.

The trench structures used for the experiments are typically less than $1 \mu \mathrm{m}$ deep and have various aspect ratios. The trenches are typically made of etched $\mathrm{SiO}_{2}$ on $\mathrm{Si}$. Metals that can be used for lining in ionized magnetron sputter metal deposition processes include $\mathrm{Ti}, \mathrm{TiN}, \mathrm{Ta}, \mathrm{TaN}, \mathrm{Cu}, \mathrm{Cr}, \mathrm{AlCu}$, AIN, W, and WN.

The numerical simulator used to predict film topography is called SHADE (shock-tracking algorithm for deposition and etching). It uses the shock-tracking method $^{7}$ for its surface evolution algorithm and has been applied to various etch and deposition processes. ${ }^{8-10}$ For the two-dimensional simula- 
DC Magnetron (rotating magnet, $29 \mathrm{~cm}$ dia.)

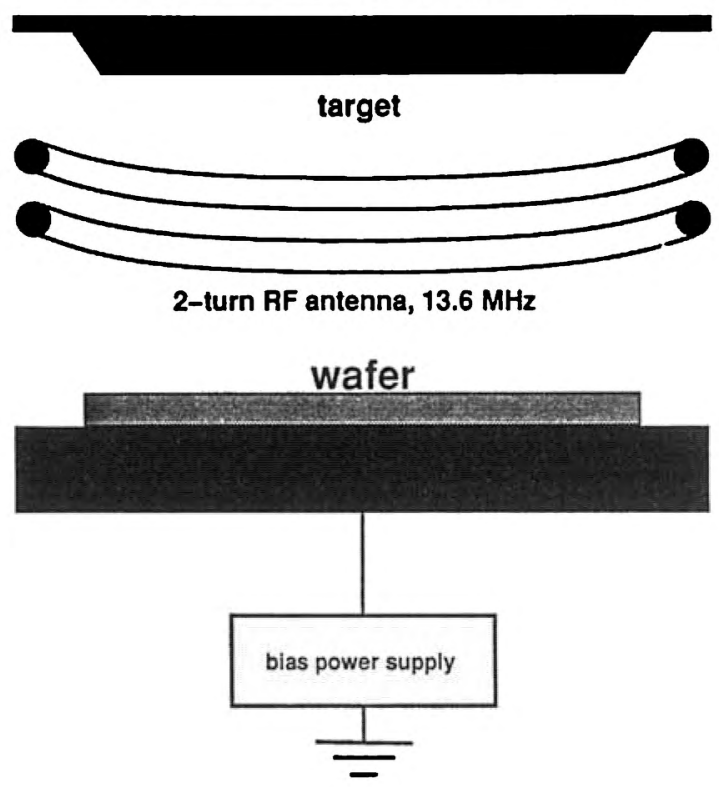

FIG. 1. Schematic diagram of the experimental apparatus.

tion, the surface is represented by a sequence of nodes (string) and the fluxes of Ar ions, metal ions, and neutral atoms are calculated at each node. Since the nodes are connected by straight line segments, there are at least two surface normals at each node: surface normals associated with left-side and right-side line segments. The shock-tracking method allows multiple surface normals at each nodal point in addition to these two surface normals. The multiple surface normals may be regarded as an approximation of the corner point (node) by an infinitesimal polygonal surface. Since the etch rate (or sputtering yield) of the material surface depends on the angle formed by the the incident ion flux and surface normal, ${ }^{\text {" }}$ the multiple surface-normal representation provides an accurate description of surface evolution. Details of the shock-tracking method may be found in Ref. 7 .

Figure 2 schematically shows the transport of neutral and ion species near the surface. Since the length scales of the trench are sufficiently smaller than the mean free paths, collisionless transport is a valid assumption for all the gas-phase species. As indicated in Fig. 2, the metal and Ar ion fluxes are assumed to be unidirectional and vertical to the substrate top surface. Since the value of the ion temperature measured in electron volts is sufficiently smaller than the bias voltage, the angular distribution of the ions is insignificant. On the other hand, the neutral metal flux has a large angular distribution. For the sake of simplicity, we assume that the incident neutral metal has an uniform angular distribution with cutoff angle $\Theta$. For a low-pressure operation, where ions sputtered from the target cathode in the magnetron region undergo virtually no collisions, this cutoff angle is determined by the tool geometry, i.e., the ratio of the distance between the target and wafer to the radius of the target. ${ }^{10}$

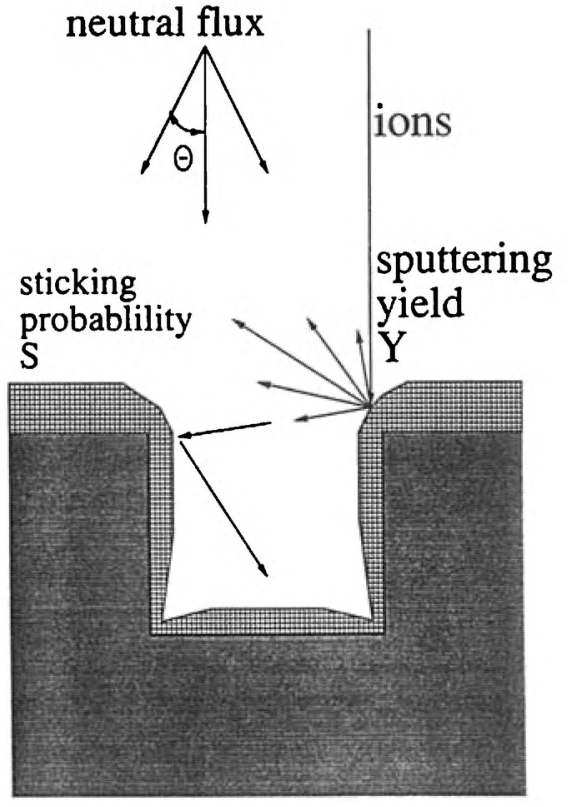

FIG. 2. Schematic diagram of particle transport.

Here we chose $\Theta=52^{\circ}$ based on our tool geometry ${ }^{10}$ for the simulation presented in this article.

When an energetic ion hits the surface, it sputters the surface material with sputtering yield $Y$. The sputtering yield, which is defined as the number of ions sputtered from the surface per incident ion, depends on the angle $\theta$ formed by the incident flux and surface normal. Figure 3 shows the angle dependence of the etch rate [which is proportional to $Y(\theta) \cos \theta]$ that is used for the numerical simulation. The rate is normalized to one at $\theta=0$. The film topographies ob-

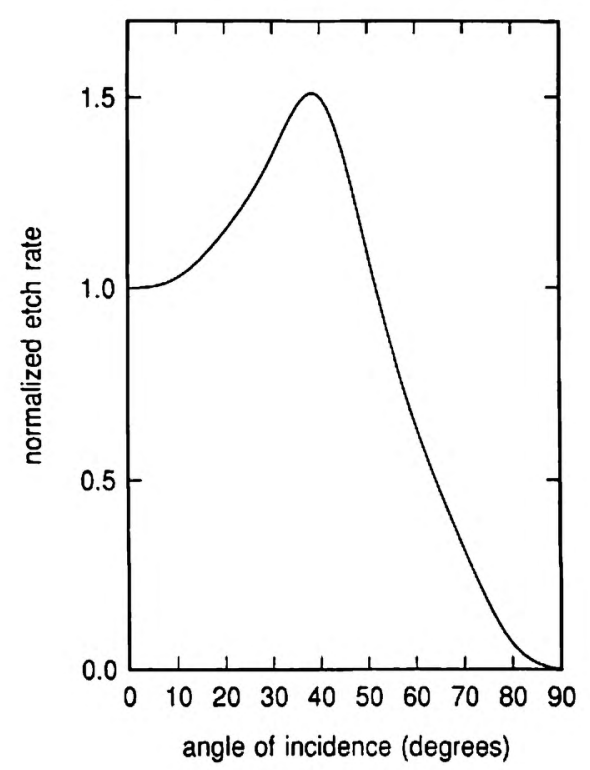

FIG. 3. The dependence of the normalized etch rate as a function of the slope angle (i.e., angle of ion beam incidence) used for the simulation. 
tained from the numerical simulation with this etch rate function are in good agreement with experimental observations, as will be shown later.

Metal atoms sputtered by the incident ions are assumed to have sufficiently low energies so that they do not resputter the surface material when then reach other surface sites. Instead, such atoms and the neutral metal atom flux directly coming from the plasma source region are adsorbed on the surface. The probability that an adsorbed atom stays on the surface indefinitely is called the sticking coefficient $\mathscr{Y}$. For $\mathrm{Cu} . \mathscr{\mathscr { Y }}=1$ is known to be a good approximation. Those atoms that are reemitted (i.e., desorbed or resputtered) from the surface are assumed to have the cosine angular distribution about the surface normal:

$$
f^{R}=f_{\nu}(|\mathbf{v}|, \mathbf{x}) \cos ^{\nu+1} \theta
$$

with $\nu=0$, where $f^{R}$ is the velocity distribution function of the reemitted atoms at position $\mathbf{x}$ on the boundary curve, and $\theta$ is the angle between the velocity vector $\mathbf{v}$ and the surface normal $\hat{\mathbf{n}}$ [Fig. 4(a)]. If $\nu>0$ or $-1<\nu<0$, the function above represents over-cosine or under-cosine distributions, respectively.

The normal component of the incoming flux at position $\mathbf{X}$ on the surface is related to the velocity distribution function $f(\mathbf{v}, \mathbf{X})$ as

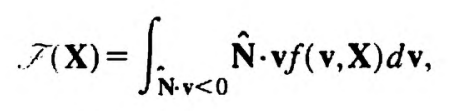

where $\hat{\mathbf{N}}$ is the unit vector normal to the surface at $\mathbf{X}$, and the integration is taken over the half velocity space $(\hat{\mathbf{N}} \cdot \mathbf{v}<0)$. The outgoing flux is defined in a similar manner with the integration over the other half space $\hat{\mathbf{N}} \cdot \mathbf{v}>0$.

The normal component of the outgoing flux from the surface. i.e., the flux of reemitted metal atoms, is the sum of the desorption flux and resputtering flux:

$$
\text { Tout } \left.^{\text {ou }}\right)=(1-\mathscr{C}) \mathscr{F}^{\text {in }}(\mathbf{X})+\mathscr{F}^{\mathrm{etch}}(\mathbf{X})
$$

where $\mathscr{F}^{\text {in }}(\mathbf{X})$ is the incoming flux of (low-energy) metal atoms and therefore the first term $(1-\mathscr{C}) \mathscr{F}^{\text {in }}$ represents the total desorption flux. The second term represents the resputtering flux due to the ion bombardment (etching). The incoming neutral metal flux $\mathscr{F}^{\text {in }}(\mathbf{X})$ may be split into the two components

$$
\mathscr{7}^{\mathrm{in}}(\mathbf{X})=\mathscr{F}^{k}(\mathbf{X})+\mathscr{F}^{\mathbb{D}}(\mathbf{X})
$$

where the first term $\mathscr{F}^{R}$ represents the incoming neutral metal flux that is originated (reemitted) from other sites on the surface and the second term $\mathscr{F}^{D}$ represents the neutral metal flux arriving directly from the plasma source. Using the reemission distribution function Eq. (1), we may relate the desorption flux $\mathscr{F}^{R}(\mathbf{X})$ to the outgoing metal atom flux $\mathcal{F}^{\mathrm{oll}}(\mathbf{x})$ at every position $\mathbf{x}$ on the surface as

$$
\mathscr{F}^{R}(\mathbf{X})=\frac{A_{\nu+4}(\nu+2)}{\pi} \int_{\mathscr{A}} d s K_{\nu}(\mathbf{X}, \mathbf{x}) \mathscr{F}^{\text {out }}(\mathbf{x}),
$$

where

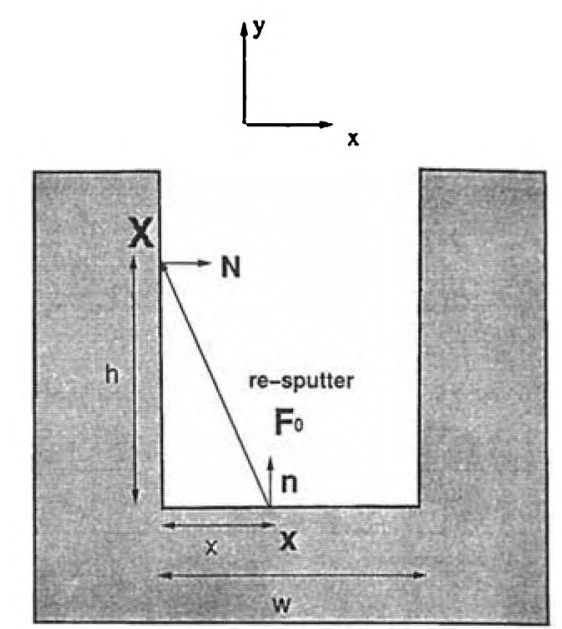

(a)

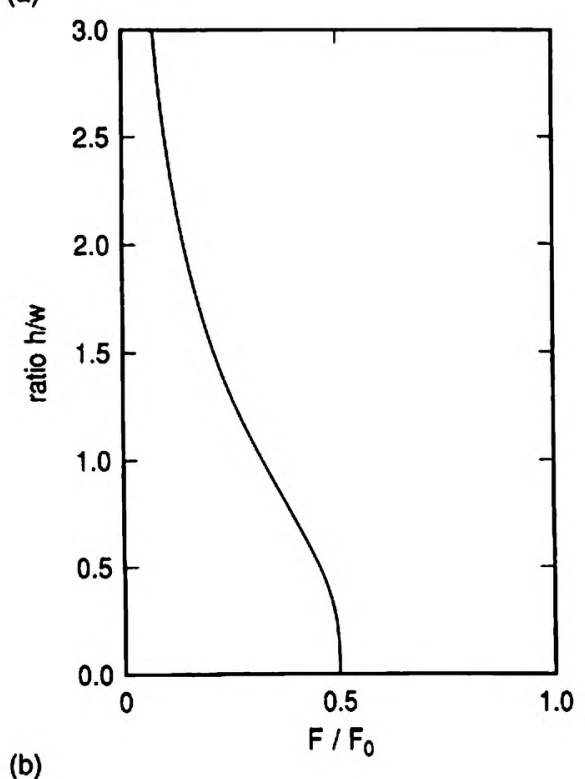

(b)

FiG. 4. (a) The coordinate system. (b) The flux ratio $\mathscr{F} / \mathcal{F}_{0}$ (which is proportional to the film thickness deposited by resputtering) as a function of the normalized height $h / w$ of the trench wall.

$$
K_{\nu}(\mathbf{X}, \mathbf{x})=g(\mathbf{X}, \mathbf{x}) \frac{(\hat{\mathbf{R}} \cdot \hat{\mathbf{N}})|\hat{\mathbf{R}} \cdot \hat{\mathbf{n}}|^{\nu+1}}{R}
$$

is the integration kernel, the coefficient $A_{\nu+4}$ is given by

$$
A_{\nu+4}=\Gamma\left(\frac{\nu+3}{2}\right) \Gamma\left(\frac{1}{2}\right) / 2 \Gamma\left(\frac{\nu}{2}+2\right) \text {, }
$$

and $\mathcal{\beta}$ denotes the surface boundary. Note that for the cosine distribution ( $\nu=0), A_{4}=\pi / 4$. In Eq. (5), the function $g$ is the visibility factor (i.e., $g=1$ if the points $\mathbf{X}$ and $\mathbf{X}$ are on the line of sight, and $g=0$ otherwise), $\mathbf{R}=\mathbf{x}-\mathbf{X}, R=|\mathbf{R}|, \hat{\mathbf{R}}=\mathbf{R} /$ $R$, and $\hat{\mathbf{n}}$ is the unit vector normal to the surface at $\mathbf{X}$.

Substituting Eqs. (2) and (3) into Eq. (4) results in an integral equation for $\mathscr{P}^{R}(\mathbf{X})$ for given source terms $\mathscr{F}^{\text {ech }}(\mathbf{X})$ and $\mathscr{F}^{D}(\mathbf{X})$. More details of the gas-phase transport, including the derivation of Eq. (4) and actual expressions for the source terms $\mathscr{F}^{\text {etch }}(\mathbf{X})$ and $\mathscr{F}^{D}(\mathbf{X})$, may be found in Ref. 10. 


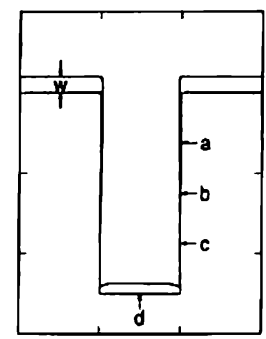

(a) $Y=0.0$

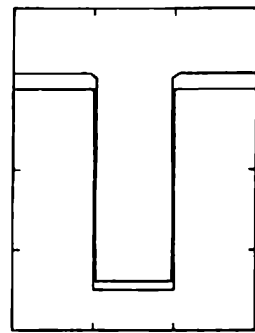

(b) $Y=0.4$

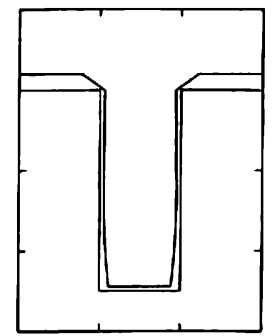

(c) $Y=1.0$
Fig. 5. Profiles of the deposited films obtained from numerical simulations. The aspect ratio of each trench is 2.5 . The ratio of the ion to neutral flux on the top surface is $1: 1$. The sputtering yields at zero incident angle $Y(0)$ are (a) 0 , (b) 0.4 , and (c) 1.0 .

\section{NUMERICAL AND EXPERIMENTAL RESULTS}

Before proceeding to numerical simulations, we briefly discuss resputtering on trench bottoms. To estimate the amount of resputtering from a trench bottom, we first ignore sidewall metal deposition due to direct neutral fluxes from the plasma source. In this case, as shown in Fig. 4(a), the flux received at height $h$ on the sidewall is only related to the resputtered outgoing flux $\mathscr{F}_{0}$ from the bottom through Eq. (4). The resputtering flux $\mathscr{F}_{0}$ is assumed to be uniform over the entire trench bottom. Assuming that the reemission has the cosine distribution [i.e., $\nu=0$ in Eq. (1)], we obtain from Eq. (4),

$$
\begin{aligned}
\mathscr{F} & =\frac{\mathscr{F}_{0}}{2} \int_{0}^{w} d x \frac{(\hat{\mathbf{R}} \cdot \hat{\mathbf{N}})|\hat{\mathbf{R}} \cdot \hat{\mathbf{n}}|}{R} \\
& =\frac{\mathscr{F}_{0}}{2} \int_{0}^{w} d x \frac{h x}{\left(\sqrt{x^{2}+h^{2}}\right)^{3}} \\
& =\frac{\mathscr{F}_{0}}{2}\left(1-\frac{h}{\sqrt{w^{2}+h^{2}}}\right),
\end{aligned}
$$

where we take the $\mathrm{x}$ axis along the bottom surface, and $w$ represents the width of the trench bottom. Figure 4(b) shows the resputtered flux received on a vertical sidewall as a function of the ratio $h / w$. Since the deposition rate is proportional to the flux $\mathscr{F}$, Fig. 4(b) also represents the relative thickness of the deposited thin film on the vertical sidewall.

For an infinitely wide trench (i.e., $w=\infty$, aspect ratio is 0 ), the magnitude of the resputtered flux becomes independent of the height $h$ and is given by $\mathscr{F}=\frac{1}{2} \mathscr{F}_{0}$. This is not surprising since the limit of $w \rightarrow \infty$ with fixed $h$ is equivalent of the limit of $h \rightarrow 0$ with fixed $w$. (Note that. $\mathscr{F}$ depends only on the parameter $h / w$.) As is evident in Fig. 4(b), the flux variation is small for small $h / w$. This result indicates that the smaller the aspect ratio is, the more uniformly the films are deposited on the sidewalls.

In numerical simulations that we shall present momentarily, the neutral metal flux from the source is also taken into account. Since metal atoms sputtered from the target are not generally $100 \%$ ionized by the RFI coil, the effect of the neutral flux cannot be ignored. In typical operations, the ratio

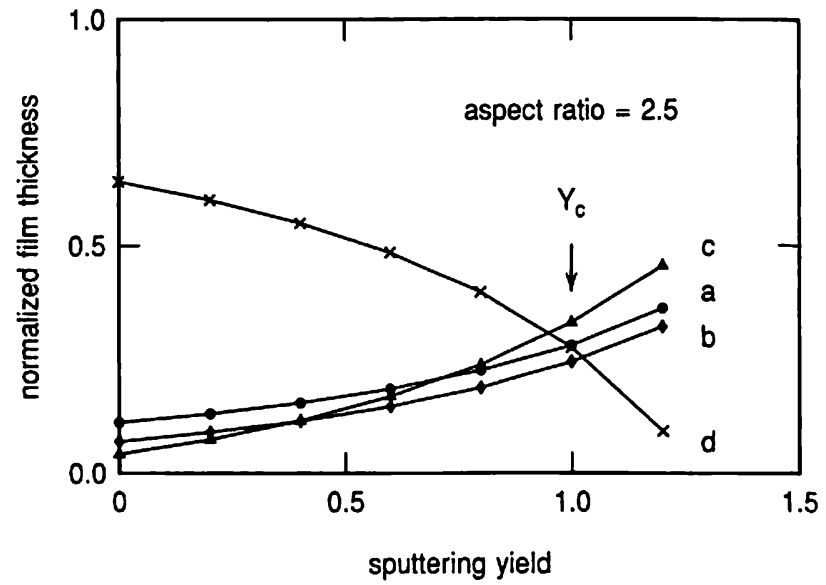

FiG. 6. The ratio of film thickness at each position $[a, b, c$, and $d$ indicated in Fig. 5(a)] to the top film thickness $w$ as a function of the sputtering yield $Y(0)$ under the conditions used in Fig. 5.

of the metal neutral flux to the metal ion flux is about $1: 1 .^{10}$ As mentioned earlier, the metal neutral flux is assumed to have an isotropic distribution with a cutoff angle $52^{\circ}$. The ion flux is a delta-function beam, perpendicularly incident upon the horizontal substrate surface.

Figure 5 shows profiles of deposited thin metal films (liners) under three different sputtering yields $Y=Y(0)$ at angle 0 (horizontal surface); (a) $Y=0$, (b) 0.4 , and (c) 1.0. The aspect ratio of the initial trench is 2.5 and the sticking coefficient $\mathscr{Y}$ is assumed to be one. The value $Y$ is actually the effective sputtering yield, i.e., the number of sputtered atoms per incident metal ion, regardless of whether the sputtering is due to metal ions or $\mathrm{Ar}$ ions. If sputtering by the $\mathrm{Ar}$ ion flux is negligible, then $Y$ is the $\mathrm{Cu}$-on-Cu sputtering yield.

Figure 6 shows the step coverage (i.e., ratio of the film thickness at a particular position to the flat-surface film thickness $w$ ) at four different sites indicated in Fig. 5(a) as functions of the effective sputtering yield $Y$. The positions a, b, c are at $\frac{1}{4}, \frac{1}{2}$, and $\frac{3}{4}$ of the trench depth, and $d$ is at the center of the trench bottom. Since the ion to neutral metal flux ratio is $1: 1$, the film is deposited on the top surface only when $Y<2.0$.

As may be seen in Fig. 5(c), the sidewall film thickness is not necessarily a monotonic function of the height, unlike the case of Fig. 4(b). At position a, a significant portion of the incoming metal flux arrives directly from the plasma source, rather than the resputtering from the bottom, and thus the film thickness is slightly larger than that at position $b$. When the sputtering yield $Y$ is high [e.g., Fig. 5(c)], a significant portion of the incoming metal flux at position $\mathrm{c}$ is due to the resputtering from the trench bottom, and thus the film thickness is again slightly larger than that at position $b$. Therefore, the film topography on the sidewall tends to be slightly bowed (thicker near the top and bottom and thinner in the middle) when $Y$ is high. However, for sufficiently thin films, such as those used in liner technology, this variation of the sidewall-film thickness is not significant.

The critical sputtering yield $Y_{c}$, which we define as the $Y$ 


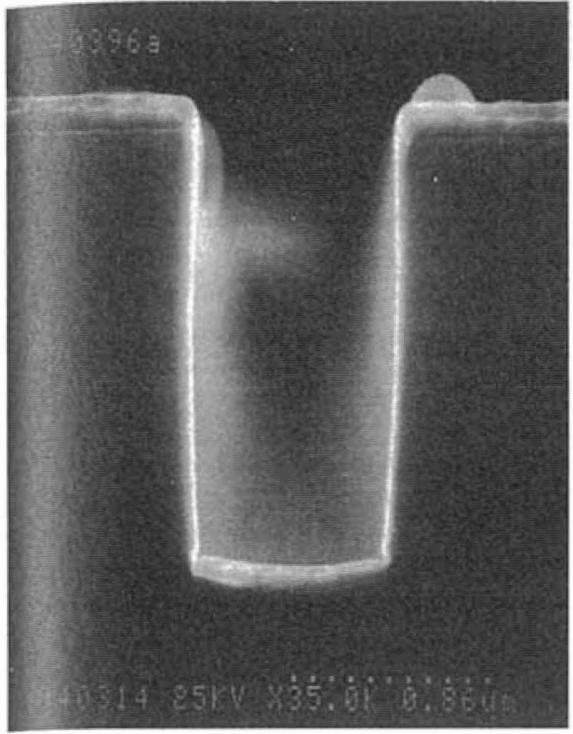

(a)

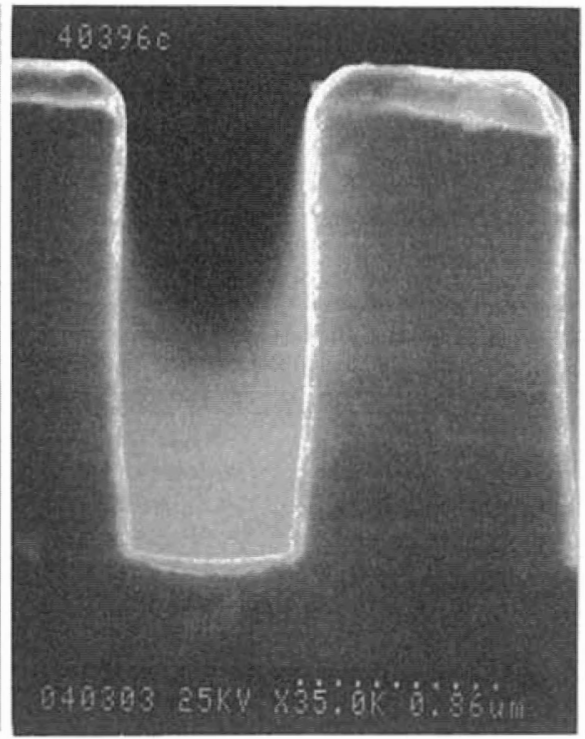

(b)

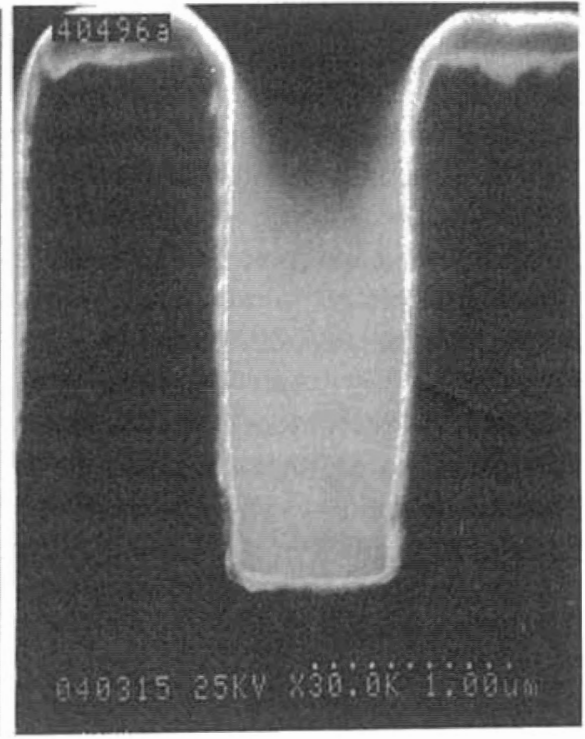

(c)

FIG. 7. SEM pictures of $\mathrm{Cu}$ deposition under the conditions similar $\mathrm{to}$ Fig. 5 . The applied bias voltages are (a) 12 , (b) 50 , and (c) $100 \mathrm{~V}$.

value at which the step coverages at $b$ (middle of the sidewall) and $d$ (middle of the bottom) are equal, gives a good guidance for the film conformality. Under the conditions used in Fig. 5, $Y_{c}=1.0$.

Experiments were done with $\mathrm{Ti}, \mathrm{TiN}, \mathrm{AlCu}$, and $\mathrm{Cu}$ over the range of $0.5-4$ aspect ratios and good conformal step coverages were obtained in all these cases. Figure 7 shows typical results of such systems under conditions similar to those used in Fig. 5. The bias voltages used in these experiments were 12, 50, and $100 \mathrm{~V}$ for Figs. 7(a), 7(b), and 7(c), respectively. $\mathrm{Cu}$ films are shown in Fig. 7 since the higher brightness of $\mathrm{Cu}$ in scanning electron microscopy (SEM) photographs most clearly delineates the film topography.

Figure 8 shows simulation results of liner deposition on trenches of aspect ratio 1 . The conditions used here are the same as those used in Fig. 5. Figure 9 shows the step coverage (i.e., normalized film thickness) at four different positions indicated in Fig. 8(a) as functions of the sputtering yield $Y$. The positions a, b, c are at $1 / 4,1 / 2$, and $3 / 4$, respectively, of the trench depth, and $d$ is at the center of the trench bottom, as before. It is seen in Fig. 9 that the critical sput-

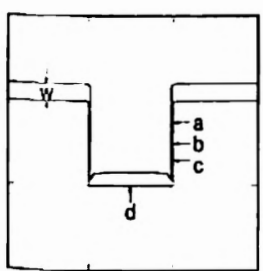

(a) $Y=0.0$

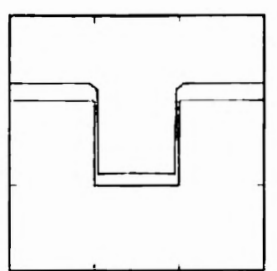

(b) $Y=0.4$

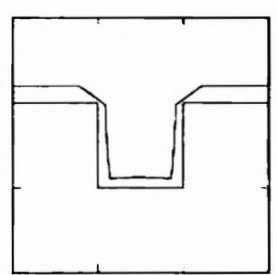

(c) $Y=1.0$
Fic. S. Profiles of the deposited films obtained from numerical simulations. The aspect ratio of each trench is 1 . Other conditions are the same as those used in Fig. 5. The sputtering yields at zero incident angle $Y(0)$ are (a) 0 . (b) 0.4, and (c) 1.0. tering yield for conformality is again $Y_{c} \simeq 1$. Evidently, $Y_{c}$. depends weakly on the aspect ratio $A$ for the range $1 \leqslant A \leqslant 2.5$ studied here. Thus the ionized magnetron sputter metal deposition system with an appropriate sputtering yield $Y$ can form conformal metal step coverages over trenches with a wide range of aspect ratios in a single process.

\section{CONCLUDING REMARKS}

Numerical simulations together with experimental results show that conformal deposition of metal liners can be achieved by simultaneous deposition and resputtering in ionized magnetron sputter metal deposition processes. The ionized magnetron sputter deposition process has two significant advantages over the conventional magnetron sputter deposition process. One is the directionality (or anisotropy) of the

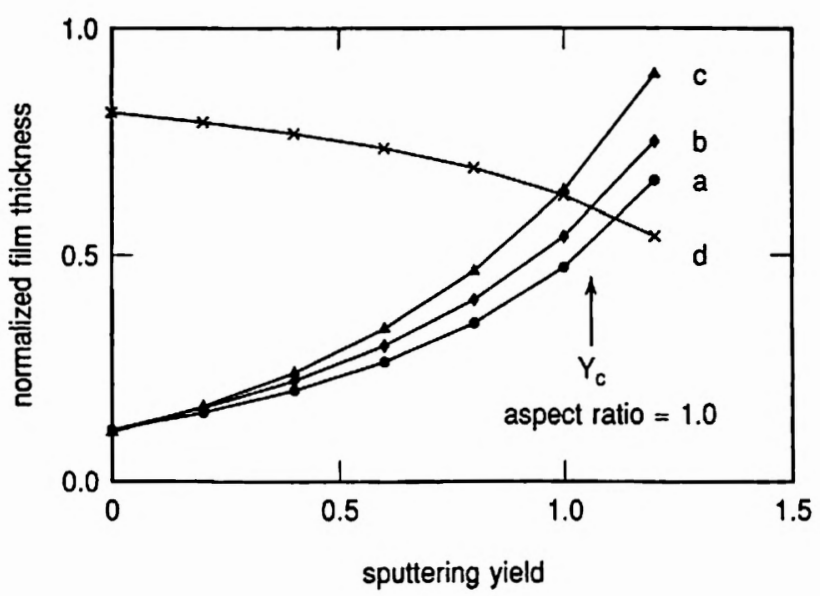

FIG. 9. The ratio of film thickness at each position [a, b, c, and d indicated in Fig. 8(a)] to the top film thickness $w$ as a function of the sputtering yield $Y(0)$ under the conditions used in Fig. 8. 
metal deposition flux due to the presence of highly ionized gas-phase metal and the applied bias voltage on the substrate surface. With high ionization of metal in the plasma region, a large metal ion flux with high directionality may be extracted from the source to the substrate without damaging the substrate. The other advantage is its ability to control the ion flux energy incident upon the substrate surface. The amount of resputtering from the substrate surface can be easily controlled by the bias voltage.

It is also shown that, by selecting an appropriate bias voltage to control surface resputtering, we are able to form conformal step coverages over various trenches with different aspect ratios in a single process. In this method, metal atoms resputtered from the bottom of the trench are effectively redeposited on trench sidewalls. As the bias voltage (i.e., sputtering yield on the sample surface) is increased, the film thickness on the sidewalls increases, whereas the film thickness on the trench bottom decreases. The appropriate bias voltage (i.e., critical sputtering yield) is the one that gives similar film thicknesses on sidewalls and trench bot- tom. Since the critical sputtering yield $Y_{c}$ is almost independent of trench aspect ratios, one can conformally line trenches with a wide variety of aspect ratios in a single process.

'A. V. Gelatos, A. Jain, R. Marsh, and C. J. Mogab, MRS Bull. 19, 49 (1994).

${ }^{2}$ S. M. Rossnagel, D. Mikalsea, H. Kinoshita, and J. J. Cuomo, J. Vac. Sci. Technol. A 9, 261 (1991).

${ }^{3}$ S. K. Dew, D. Liu, M. J. Brett, and T. Smy, J. Vac. Sci. Technol. B 11, 1281 (1993).

${ }^{4} \mathrm{R}$. Joshi and S. Brodsky, Proceedings of the 1992 VLSI Multilevel Interconnect Conference, June 1992 (unpublished), p. 235.

${ }^{5}$ S. M. Rossnagel and J. Hopwood, Appl. Phys. Lett. 63, 3285 (1993).

${ }^{6} S$. M. Rossnagel and J. Hopwood, J. Vac. Sci. Technol. B 12, 449 (1994).

${ }^{7}$ S. Hamaguchi, M. Dalvie, R. T. Farouki, and S. Sethuraman, J. Appl. Phys. 74, 5172 (1993).

${ }^{8}$ S. Hamaguchi and M. Dalvie, J. Vac. Sci. Technol. A 12, 2745 (1994).

'S. Hamaguchi and M. Dalvie, J. Electrochem. Soc. 141, 1964 (1994).

${ }^{10}$ S. Hamaguchi and S. M. Rossnagel, J. Vac. Sci. Technol. B 13, 183 (1995).

"P. Zalm. Surf. Interface Anal. 11, I (1988). 